\title{
Volar versus Dorsal surgical approaches in fractures of proximal radius in adults: A prospective randomized controlled trial
}

\author{
Rijal N', Shrestha BP2 , Kalawar RPS ${ }^{3}$, Pokharel B ${ }^{3}$, Baral P4
}

${ }^{1}$ Nishchal Rijal, Consultant Orthopaedic Surgeon, Dadeldhura Hospital, Dadeldhura, Nepal; ${ }^{2}$ Bikram Prasad Shrestha, Professor; ${ }^{3}$ Rosan P. S. Kalawar, Associate Professor; ${ }^{3}$ Bishnu Pokharel, Associate Professor; ${ }^{4}$ Pramod Baral, Assistant Professor; Department of Orthopaedics, B. P. Koirala Institute of Health Sciences (BPKIHS), Dharan, Nepal.

\begin{abstract}
Background: Open reduction and internal fixation with plates and screws is the standard treatment for both-bone forearm fractures in adults. For fractures of the proximal one-third or half of the radius, both the volar (Henry) and dorsal (Thompson) approaches are employed and each one has its own advantages and disadvantages.

Objectives: The aim of the study was to compare the functional outcome, intraoperative parameters, complications and duration of union between the two approaches.

Methodology: The randomized controlled trial was conducted in the Department of Orthopaedics, B. P. Koirala Institute of Health Sciences (BPKIHS), Dharan, over a period of 12 months (October 2017 to September 2018). Fifty patients with proximal radius fractures were randomized into two groups: Group A and Group B. Patients in Group A ( $n=25)$ underwent surgery via the volar approach whereas those in Group B $(n=25)$ via the dorsal approach. They were evaluated on the second postoperative day and at 2, 6, 12 and 24 weeks postoperatively with regards to functional outcome, clinical and radiologic signs of fracture union and complications.

Results: The difference in the intraoperative parameters (length of incision, duration of surgery, tourniquet time) was not statistically significant between the two groups ( $p$-values $>0.05$ ). There was a progressive increase in range of motion $(\mathrm{ROM})$ and a progressive decrease in the Disabilities of the Arm, Shoulder and Hand (DASH) scores, signifying improving functional outcome in both the groups during patient follow-up ( $p$-values $>0.05$ ). Three cases of posterior interosseous nerve (PIN) palsies (one in the volar group and two in the dorsal group), one case of deep surgical site infection in the volar group and one case of nonunion in the dorsal group were encountered.

Conclusion: The surgical approaches were comparable in terms of functional outcome, intraoperative parameters, complications and time to union. Though cases with PIN palsy were seen in both the groups, the difference in the occurrences was not statistically significant. As both the approaches were associated with a low rate of complications, either can be used based on the preference of the operating surgeon. However, large scale multicenter studies are necessary to recommend guidelines on the choice of surgical approach for proximal radius fractures in adults.
\end{abstract}

Key words: Dorsal approach; Fracture proximal radius; Henry; Thompson; Volar approach

DOI: https://doi.org/10.3126/jkmc.v8i4.32388

\section{INTRODUCTION}

$\mathrm{F}$ ractures of both bones of the forearm are relatively common injuries. Proximal radius fractures account for $74.3 \%$ of all proximal forearm fractures ${ }^{1}$. They usually occur in young males with good bone stock, most frequently in the setting of high-energy trauma such as motor vehicle accidents or sports injuries ${ }^{2}$.

Address for correspondence

Dr. Nishchal Rijal

Consultant Orthopaedic Surgeon

Dadeldhura Hospital, Dadeldhura, Nepal

Email: nishchalrj@gmail.com

ORCID: https://orcid.org/0000-0001-5948-6197
The relationship between radius and ulna is critical for function, especially pronation and supination; hence the forearm has been called a 'functional joint'. Thus, it is important to re-establish length and rotation for the forearm to maintain its dynamic function ${ }^{3}$.

Treatment by closed reduction and cast immobilization results in poor functional outcome with unsatisfactory results, usually as a consequence of malunion, nonunion or synostosis 4 . Therefore, open reduction and internal fixation (ORIF) with plates and screws is the standard treatment for both-bone forearm fractures in adults ${ }^{5-10}$. 
The surgical approach is generally selected according to the level of fracture and soft tissue damage ${ }^{11}$. For the proximal one-third or half of the radius, both the volar and dorsal approaches are employed and each one has its own advantages and disadvantages ${ }^{2-4}$.

The volar approach to the proximal radius was first described by Henry in $1927^{12}$. It allows easy distal extension of exposure with adequate soft tissue coverage, providing access to almost the whole length of the bone. However, impingement on the bicipital tuberosity and biceps tendon, in addition to plate positioning on the compression side of the bone, make this approach less desirable ${ }^{12}$. The posterior interosseous nerve (PIN) may be at risk, especially during retraction of the supinator, causing a neurapraxia. When the nerve lies posteriorly juxtaposed to the radial neck, which occurs in a quarter of the population, retractor placement can compress it against the bone ${ }^{13}$. Nonetheless, fixation to the level of the tuberosity can be safely accomplished without an increase in risk to the $\mathrm{PIN}^{14}$.

On the other hand, the dorsal approach was first described by Thompson in $1918^{15}$. It is traditionally recommended for proximal radius fractures because of theoretically improved exposure and plating being done on the tensile surface ${ }^{13}$. The PIN can be visualized and protected using this approach; however, it is at risk of being irritated by the plate. Likewise, plate removal by this approach is not recommended as scarring caused by the previous surgery may make visualization of the PIN more difficult ${ }^{16}$. Furthermore, when the PIN is adjacent to the radial neck, it is at risk of entrapment under a plate ${ }^{17}$.

Some studies have concluded that there is no significant difference in the functional outcome of the two approaches ${ }^{14,18,19}$. Similarly, some studies have given preference to the volar approach ${ }^{16,20-23}$ and others, the dorsal approach ${ }^{11,24}$. So the purpose of this study was to compare these two approaches with respect to the functional outcome as per the Disabilities of the Arm, Shoulder and Hand (DASH) score and range of motion (ROM), intraoperative parameters, incidence of PIN palsy, duration of union and other complications, so that we have better evidence and clear guidelines for choosing the method of surgical exposure for proximal radius fractures in adults.

\section{METHODOLOGY}

This randomized controlled trial was conducted in the Department of Orthopaedics, B. P. Koirala Institute of Health Sciences (BPKIHS), Dharan, a tertiary care hospital in Eastern Nepal, over a period of 12 months from October 2017 to September 2018. Ethical clearance was obtained from the Institutional Review Committee (IRC) of BPKIHS. The study was carried out as per the declaration of Helsinki for experiments involving humans. All skeletally mature patients (age $\geq 16$ years) diagnosed with closed and Gustilo Grade I open fracture of the proximal half of radius with or without ulna fracture, giving written and informed consent, were included in the study. Patients with re-fractures, pathological fractures, Gustilo Grade II and III open fractures, polytrauma, fractures with compartment syndrome or distal neurovascular deficit (DNVD) and those with associated diseases that interfered with rehabilitation, were excluded from the study. Initially, 57 patients were assessed for eligibility for our study, out of which seven patients not fulfilling the inclusion criteria were excluded. A total of 50 patients were randomized into the two groups: Group A and Group B.

On the basis of the literature study of Nasab SAM et $\mathrm{al}^{19}$, who treated proximal radius fractures using volar approach $(n=39)$ and dorsal approach $(n=31)$, based on forearm rotation, the sample size was calculated as 50 with 25 patients in each group. Patients presenting to the Emergency Room (ER) and Outpatient Department (OPD) of BPKIHS, Dharan were assessed for eligibility. The diagnosis was confirmed by X-rays [Anteroposterior (AP) and lateral views] and the affected limb was splinted and analgesics were administered. An informed and written consent for the study was taken from each patient after explaining about the procedure, complications and possible outcomes. Then the relevant preoperative investigations were ordered. Once the patients were cleared for surgery after pre-anaesthetic assessment, they were posted for elective surgery. Preoperative antibiotic (second generation cephalosporin) was administered in all cases within 30 minutes prior to surgical incision. After adequate and appropriate anaesthesia, the patient was positioned in supine position and a pneumatic tourniquet was applied over the upper arm. Further surgical approach varied according to the two groups, into which the patients were randomized according to Excel random number generation technique.

1. Group A: Volar approach group (Henry)

2. Group B: Dorsal approach group (Thompson)

The surgeries were performed by the senior consultants at the institute. All the intraoperative parameters were recorded. Postoperatively, arm pouch sling/ above elbow plaster of Paris (POP) slab (in 90 degrees of elbow flexion and neutral forearm rotation) was applied 
according to the type of fixation. Check X-rays were assessed. Immediate postoperative complications e.g. DNVD, if any, and severity of pain [Visual Analog Scale (VAS) scores] were noted. The wound was inspected on the second postoperative day and the patient was discharged when found to be satisfactory.

The patients were followed up in OPD at 2, 6, 12 and 24 weeks postoperatively with check X-rays at each visit except at 2 weeks. The sutures were inspected and removed at the first follow-up visit on the $14^{\text {th }}$ postoperative day, if healthy. The functional outcome, $\mathrm{ROM}$, clinical and radiologic signs of fracture union and complications were assessed. The patients were put through ROM exercises of elbow and forearm within the limits of pain.

Fracture healing was determined as fracture bridging seen on at least three out of four cortices on AP and lateral radiographs. Union was assessed based on the criteria of Anderson et al. ${ }^{10}$, which classified fractures healing within 6 months of surgery as unions, those healing after 6 months without additional intervention as delayed unions, and those that failed to heal after 6 months or required additional unplanned surgical intervention to achieve healing as nonunions ${ }^{10}$.

The data thus obtained were collected, checked and entered in Statistical Product and Service Solutions (SPSS) software sheet version 20 and $p$-value $<0.05$ was considered statistically significant. Proportion, measure of central tendency and dispersion of continuous variables were tested by appropriate parametric and non-parametric statistical techniques (e.g. Independent Sample T-test or Mann-Whitney U test). Chi-square test and Fisher's exact test were used for categorical data like socio-demographic variables with graphs. The outcomes at various follow-up intervals were compared between the two groups and both magnitude and significance of the difference were measured using appropriate tests. The results were compared with other relevant studies in the literature and consensus view presented comparing outcomes and complication rates between the two groups.

\section{RESULTS}

In our study, four patients were lost to follow-up after 2 weeks (two from each group). At final follow-up (24 weeks postoperatively), a total of 46 patients were analyzed (23 in each group). Since the number of patients lost to follow-up was less than $10 \%$ (i.e. five for a sample size of 50 patients), it did not significantly affect the validity of our study. (Figure 1)
The majority of the participants in this study were males (33 patients, 66\%). The mean age was 38.16 years (S.D. 13.03 years) in Group A (range: 16-66 years) and 35.16 years (S.D. 12.77 years) in Group B (range: 16-61 years). The majority of the fractures occurred on the left side (36 patients, $72 \%$ ). The most common mode of injury was fall injury, with 34 patients (68\%) presenting with fall either from height (15 patients, 30\%) or on level ground (19 patients, 38\%). The majority of the patients i.e. 37 (74\%), had fractures of both the bones. Seven patients had Gustilo grade I open fractures and among them, only three received treatments for the wound, which consisted of intravenous antibiotics and limb splintage, prior to presentation to our institute. The mean duration from injury to surgery in Group A was 5.64 days (S.D. 6.46 days) and in Group B was 7.52 days (S.D. 10.03 days). All of these variables were comparable between the two groups suggesting successful randomization and no selection bias (Table 1).

The type of anaesthesia, duration of surgery, tourniquet time, length of incision and type of implant used were comparable between the groups and their differences were not statistically significant (Table 2). The duration of surgery and tourniquet time were less in patients with isolated radius fractures as compared to those with fractures of both radius and ulna. However, these variables were not analyzed separately in our study because of the similar distribution of isolated radius fractures and both bone forearm fractures in both the groups. The average VAS score for pain in the immediate postoperative period was $8.02 \pm 0.6$ in Group A and 8.28 \pm 0.54 in Group B. Three patients developed PIN palsies after the surgery; one in Group A and two in Group B. Only one patient had postoperative wound infection in Group A (Table 3).

There was a progressive increase in the ROM (wrist flexion-extension, forearm supination-pronation and elbow flexion-extension) (Table 4) and a progressive decrease in the DASH scores in both the groups (Table 5). These results indicated improving functional outcomes that were not significantly different.

There was one patient in Group B that did not show radiologic signs of union at 6 months (Table 6). The remaining 45 patients showed union by the last followup with the average time to union being $15.91 \pm 6.16$ weeks in Group A and $15 \pm 6.61$ weeks in Group B (Table 7, Figures 2 and 3). 


\section{CONSORT Flow Diagram}

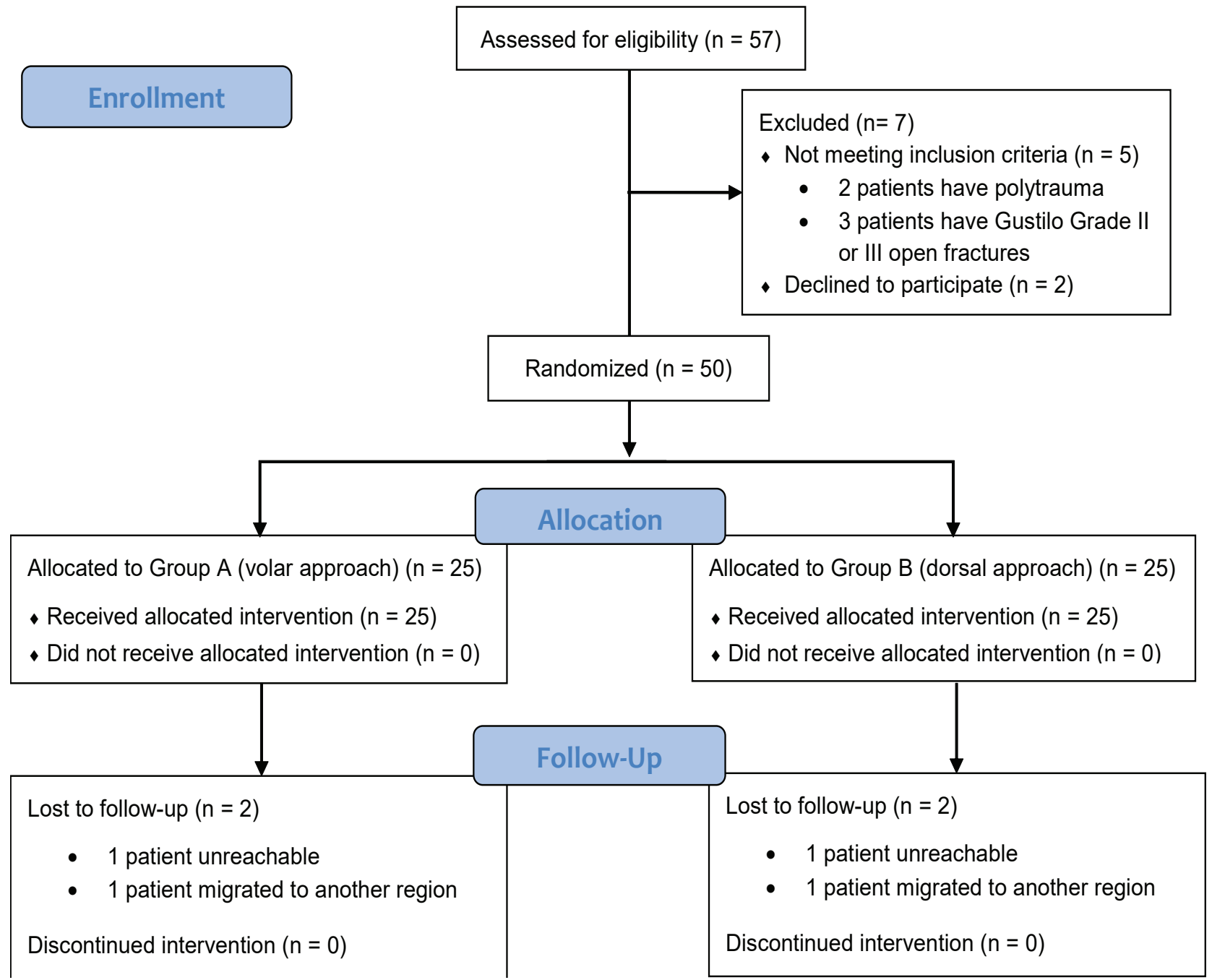

Figure 1: CONSORT flow diagram of our study

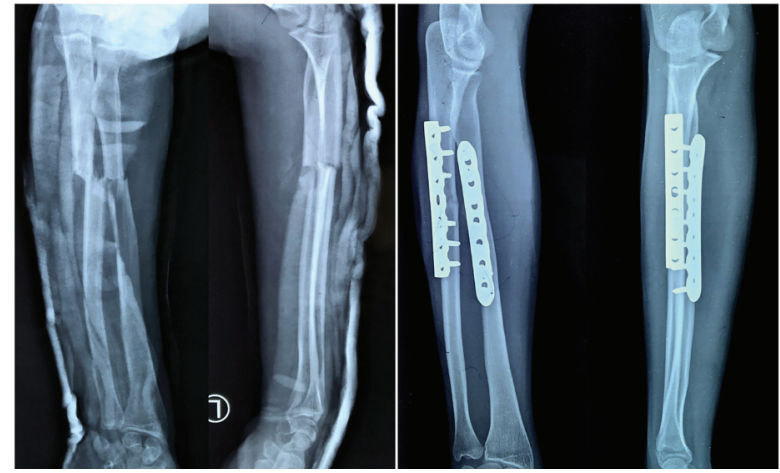

Figure 2: Group A: X-rays - preoperative and at final follow-up (AP and lateral views)

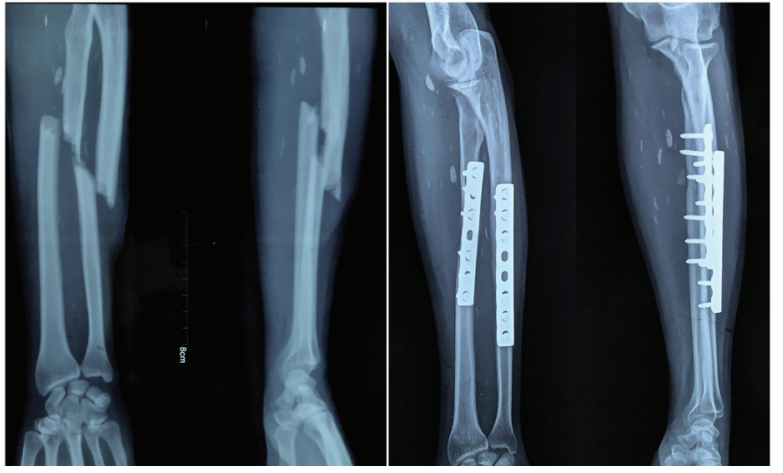

Figure 3: Group B: X-rays - preoperative and at final follow-up (AP and lateral views) 
Rijal $\mathrm{N}$ et al.

Table 1: Socio-demographic profile of the patients

\begin{tabular}{|c|c|c|c|c|}
\hline \multirow{2}{*}{ Parameters } & & \multicolumn{2}{|c|}{ Group } & \multirow{2}{*}{ p-value } \\
\hline & & Group A (n=25) & Group B $(n=25)$ & \\
\hline \multirow{2}{*}{ Gender distribution } & Male & 18 & 15 & \multirow{2}{*}{0.370} \\
\hline & Female & 7 & 10 & \\
\hline Age in years (Mean \pm S.D.) & & $38.16 \pm 13.03$ & $35.16 \pm 12.77$ & 0.415 \\
\hline \multirow{2}{*}{ Side distribution } & Right & 7 & 7 & \multirow{2}{*}{1.000} \\
\hline & Left & 18 & 18 & \\
\hline \multirow{3}{*}{ Mode of injury } & RTA & 9 & 7 & \multirow{3}{*}{0.339} \\
\hline & Fall from height & 9 & 6 & \\
\hline & Fall on ground & 7 & 12 & \\
\hline \multirow[b]{2}{*}{ Number of bones fractured } & Fracture of radius only & 7 & 6 & \multirow[b]{2}{*}{0.747} \\
\hline & $\begin{array}{l}\text { Fractures of radius and } \\
\text { ulna }\end{array}$ & 18 & 19 & \\
\hline \multirow{2}{*}{ Type of fracture } & Closed & 21 & 22 & \multirow{2}{*}{1.000} \\
\hline & Open (Gustilo Grade I) & 4 & 3 & \\
\hline \multirow{2}{*}{$\begin{array}{l}\text { Pre-hospital wound } \\
\text { treatment (if any) }\end{array}$} & Yes & 1 & 2 & \multirow{2}{*}{0.486} \\
\hline & No & 3 & 1 & \\
\hline \multicolumn{2}{|c|}{ Injury to surgery time in days (Mean \pm S.D.) } & $5.64 \pm 6.46$ & $7.52 \pm 10.03$ & 0.952 \\
\hline
\end{tabular}

Table 2: Intraoperative parameters

\begin{tabular}{|c|c|c|c|c|}
\hline \multirow{2}{*}{ Parameters } & & \multicolumn{2}{|c|}{ Group } & \multirow{2}{*}{ p-value } \\
\hline & & Group A (n=25) & Group B $(n=25)$ & \\
\hline \multirow{2}{*}{ Type of anaesthesia } & Regional & 19 & 21 & \multirow{2}{*}{0.480} \\
\hline & General & 6 & 4 & \\
\hline Duration of surgery in minutes (Mean \pm S.D.) & & $85.4 \pm 14.71$ & $83.2 \pm 14.64$ & 0.599 \\
\hline Tourniquet time in minutes (Mean \pm S.D.) & & $69 \pm 11.54$ & $67.4 \pm 12.59$ & 0.642 \\
\hline Length of incision in $\mathrm{cm}$ (Mean \pm S.D.) & & $8.76 \pm 0.84$ & $8.84 \pm 0.98$ & 0.759 \\
\hline \multirow{2}{*}{ Implant } & LCDCP & 21 & 23 & \multirow{2}{*}{0.667} \\
\hline & LCLCP & 4 & 2 & \\
\hline
\end{tabular}

Table 3: Postoperative parameters

\begin{tabular}{|c|c|c|c|c|}
\hline \multirow{2}{*}{ Parameters } & & \multicolumn{2}{|c|}{ Group } & \multirow{2}{*}{ p-value } \\
\hline & & Group A ( $n=25)$ & Group B (n=25) & \\
\hline \multicolumn{2}{|c|}{ Immediate postoperative pain (VAS score) (Mean \pm S.D.) } & $8.02 \pm 0.6$ & $8.28 \pm 0.54$ & 0.115 \\
\hline \multirow{2}{*}{ PIN palsy } & Yes & 1 & 2 & \multirow{2}{*}{1.000} \\
\hline & No & 24 & 23 & \\
\hline \multirow{2}{*}{ Wound status at 2 weeks follow-up } & Healthy & 24 & 25 & \multirow{2}{*}{1.000} \\
\hline & Infected & 1 & 0 & \\
\hline
\end{tabular}


Table 4: Functional outcome (ROM)

\begin{tabular}{llcccc}
\multicolumn{1}{c}{ ROM (in degrees) } & Follow-up & \multicolumn{2}{c}{ Group } & \multicolumn{1}{c}{ p-value } \\
& duration & Group A (n=23) & Group B (n=23) & 0.948 \\
& 6 weeks & $115.86 \pm 9.96$ & $116.08 \pm 12.24$ & 0.852 \\
Wrist flexion-extension (Mean \pm S.D.) & 12 weeks & $133.69 \pm 11.79$ & $134.34 \pm 11.7$ & 0.368 \\
& 24 weeks & $147.39 \pm 11.95$ & $144.56 \pm 8.9$ & 0.454 \\
Forearm supination-pronation (Mean & 6 weeks & $126.73 \pm 11.54$ & $129.13 \pm 9.84$ & 0.927 \\
\pm S.D.) & 12 weeks & $143.69 \pm 9.19$ & $143.47 \pm 6.47$ & 0.264 \\
& 24 weeks & $158.04 \pm 5.16$ & $156.3 \pm 5.26$ & 0.295 \\
Elbow flexion-extension (Mean \pm S.D.) & 12 weeks & $121.52 \pm 8.17$ & $118.91 \pm 8.52$ & $131.3 \pm 6.77$ & 0.238 \\
& 24 weeks & $133.69 \pm 6.77$ & $140.43 \pm 4.24$ & $139.34 \pm 5.06$ & 0.434 \\
\hline
\end{tabular}

Table 5: Functional outcome (DASH score)

\begin{tabular}{|c|c|c|c|c|}
\hline & \multirow{2}{*}{ Follow-up duration } & \multicolumn{2}{|c|}{ Group } & \multirow{2}{*}{ P-value } \\
\hline & & Group A $(n=23)$ & Group B ( $n=23)$ & \\
\hline \multirow{3}{*}{ DASH score (Mean \pm S.D.) } & 6 weeks & $60.83 \pm 7.21$ & $63.76 \pm 8.81$ & 0.112 \\
\hline & 12 weeks & $27.46 \pm 12.42$ & $30.86 \pm 16.39$ & 0.227 \\
\hline & 24 weeks & $10.54 \pm 11.8$ & $14.01 \pm 15.86$ & 0.145 \\
\hline
\end{tabular}

Table 6: Postoperative complications

\begin{tabular}{cccc}
\hline Complications & Group & Group B (n=23) & p-value \\
None & Group A (n=23) & 20 \\
PIN palsy & 21 & 2 & 1.000 \\
Infection & 1 & 0 & 1 \\
\hline Nonunion & 1 & 0 & 1 \\
\hline
\end{tabular}

Table 7: Fracture union

\begin{tabular}{lccc}
\hline & & Group & \\
& Group A (n=23) & Group B (n=22) & p-value \\
Fracture union in weeks (Mean \pm S.D.) & $15.91 \pm 6.16$ & $15 \pm 6.61$ & 0.634 \\
\hline
\end{tabular}

\section{DISCUSSION}

Compression plating is the standard treatment for forearm shaft fractures in adults. Many studies recommend a dorsal Thompson approach for proximal radius fractures to allow for more proximal fixation points and to identify and protect the PIN ${ }^{11,24}$. Whereas others favour the use of the extensile volar Henry approach, using a medial release of the supinator to protect the PIN in the supinated position, while avoiding its dissection ${ }^{16,20-23}$. The purpose of this study was to compare these two approaches with respect to the functional outcome, intraoperative variables, complications and time to union.
CR Jockel et al. ${ }^{11}$ stated that the dorsal approach is more suitable for proximal radius of up to $2.6 \mathrm{~cm}$ distal to the elbow joint, so that damage to the PIN is prevented. However, in our study, comparable exposures of the proximal radius were seen in both the approaches. This was consistent with the study performed by Cross et al. ${ }^{18}$ where the two approaches did not result in a significant difference in the area exposed. Dashe J et al. ${ }^{14}$ observed that patients treated with a dorsal approach had fractures that were slightly more proximal ( $p$-value 0.0006). Still, this did not translate to more fixation proximal to the fracture, indicating no difference in exposures for satisfactory fixation in either approach ${ }^{14}$. 
In all the three cases with PIN palsies, the proximal radius had been exposed by subperiosteal elevation of the supinator without direct visualization of the PIN, and the plates had not been placed proximally up to the radial neck, ruling out entrapment of the nerve under the plate. As a period of 6 to 9 months may be required to observe recovery even in PIN neurapraxias ${ }^{13}$, the duration of our study was inadequate and all three patients had PIN palsies during the last follow-up at 6 months. In a multicenter study by Dashe $\mathrm{J}$ et al. $^{14}$, there were only three neurologic injuries in the series; two in the volar and one in the dorsal group. Nasab SAM et al. ${ }^{19}$ observed that radial nerve injury had occurred in three patients in the volar group (injury to superficial radial nerve in one and injury to PIN in two) and in two patients in the dorsal group (injury to PIN in both). And in their study as well, the difference in the results was not statistically significant. In another study by Nasab SAM et al. ${ }^{23}$, two cases out of 28 patients with forearm proximal fractures treated with the dorsal approach had PIN palsies. Spinner et al. ${ }^{24}$ studied seven patients who presented with an isolated extensor digitorum communis (EDC) paralysis (due to injury to PIN) immediately after surgery by the dorsal approach. However, they have inferred that the Thompson approach is safe as long as certain principles are followed ${ }^{24}$. Two studies conducted by Mekhail et al. ${ }^{16,22}$ also indicate that whichever the approach used, the PIN would always be at risk of injury, especially by traction. However, Bartonicek J et al. ${ }^{20}$ reported no cases of PIN palsy among the 13 patients with fractures of the proximal radius operated by the Henry approach. In a retrospective study by Kwasny et al. ${ }^{21}$, where 80 patients with proximal radius fractures had been treated by the volar approach, only two cases of incomplete injury to the superficial radial nerve were seen and spontaneous recovery occurred in both the cases.

Many studies have evaluated the association of PIN palsies with the Thompson approach and they have concluded that by carefully following the steps of the approach and gentle handling of soft tissues and bones, injury to the PIN can be avoided ${ }^{16,19,22,24}$. In the majority of the studies, the Henry approach has not been associated with PIN palsies and many have stated that the familiarity of the surgeon with the approach is the main reason for such a result ${ }^{16,20-23}$. In our study, we found patients with PIN palsies in both the approaches, however, the difference in the results was not statistically significant.

Only one patient, which was a case of closed fracture, developed postoperative wound infection (deep surgical site infection) in Group A at follow-up visit at 2 weeks and was treated with wound debridement and intravenous antibiotics. Frequent follow-up visits and wound treatment was done accordingly and the infection had resolved by the next follow-up visit at 6 weeks. In the study by Nasab SAM et al. ${ }^{19}$, one patient in each group had surgical site infection. Dashe $\mathrm{J}$ et al. ${ }^{14}$ observed in the series that three patients had a deep infection, all in the volar group. Nonetheless, the difference in these results was not statistically significant.

During follow-up, there was an increase in ROM in both the groups, which was not significantly different, although at 6 months, the ROM in Group A was slightly more than Group B (p-values > 0.05). In the study by Nasab SAM et al. ${ }^{19}$, there was a significant difference in the degree of forearm rotation at 1 month between the two groups $(86.15 \pm 15.06$ degrees in volar group and $96.29 \pm 10.56$ degrees in dorsal group, $p$-value 0.02 ). However, at subsequent visits, the differences were not significant and at final follow-up at 4 months, ROM values were comparable ( $p$-value 0.16$).{ }^{19}$ In contrast to our study, Nasab SAM et al. observed greater ROM in the dorsal group. Dashe J et al. ${ }^{14}$ found that the average arc of pronation-supination in the volar and dorsal groups was 160 degrees and 159 degrees and elbow range of motion was 5-132 degrees and 6-128 degrees, respectively ( $p$-values $>0.05$ ). Dietz et al. ${ }^{25}$ had concluded that malpositioning of the plates was more likely with the dorsal approach and that led to interference with the kinematics. However, such cases were not seen in our study as evidenced by comparable ROM results.

There was a progressive decrease in the DASH scores during patient follow-up signifying improving functional outcome. Slightly lower DASH scores in Group A were observed which correlated with the marginally better ROM seen in this group. Nevertheless, the differences in the results were not statistically significant and the functional outcome between the two groups were similar. Likewise, the presence of an ulna fracture did not influence the functional outcome in either group, similar to the observations of Dashe $\mathrm{J}$ et $\mathrm{al}^{14}$.

There was one patient in Group B with nonunion at 6 months postoperatively, as there was no evidence of healing in three subsequent radiographs taken at monthly intervals after 3 months of surgery. Nasab SAM et al. ${ }^{19}$ found one nonunion in each group by the last follow-up while Dashe J et al. ${ }^{14}$ observed nine nonunions, five in the volar and four in the dorsal group ( $p$-values $>0.05$ ). In our study, the remaining 45 patients showed union by the last follow-up and the difference in the 
average time to union was not statistically significant between the two groups; similar to the findings of Nasab SAM et al.

Our study had limitations as it had a small sample size, was done in a single institution and follow-up evaluation was also of a shorter duration, due to which the management of nonunion/ delayed union could not be assessed and the recovery and treatment of PIN palsies could not be evaluated properly. So a longer duration of study is recommended for better assessment and management of complications.

The strength of our study was that follow-up assessment of every patient was done by the same surgeon during the entire study period.

suggestions and constant guidance during the writing of this research article.

\section{REFERENCES}

1. Court-Brown CM. The epidemiology of fractures and dislocations. In: Court-Brown CM, Heckman JD, McQueen MM, Ricci WM, Tornetta P, McKee MD, editors. Rockwood and Green's fractures in adults. 8th ed: Wolters Kluwer; 2015. p. 59-106.

2. Streubel PN, Pesántez RF. Diaphyseal fractures of the radius and ulna. In: Court-Brown CM, Heckman JD, McQueen MM, Ricci WM, Tornetta P, McKee MD, editors. Rockwood and Green's fractures in adults. 8th ed: Wolters Kluwer; 2015. p. 1121-75.

3. Perez EA. Fractures of the shoulder, arm, and forearm. In: Canale ST, Beaty JH, editors. Campbell's operative orthopaedics. 12th ed: Mosby, Elsevier; 2013. p. 2887 9.

4. Meena RK, Chishti S, Singh AM, Singh A, Singh PI, Debbarma $S$. A prospective study comparing locking compression plate with limited contact dynamic compression plate for the treatment of adult diaphyseal both bone forearm fractures. IOSR journal of dental and medical sciences. 2013;3(6):38-43.

5. Chapman MW, Gordon J, Zissimos AG. Compressionplate fixation of acute fractures of the diaphyses of the radius and ulna. J Bone Joint Surg Am. 1989;71(2):15969.

6. Matejčić A, Mihovil I, Tomljenović M, Krolo I. Forearm shaft fractures: results of ten-year follow-up. Acta Clin Croat. 2000;39(3):147-53.

7. Droll KP, Perna P, Potter J, Harniman E, Schemitsch $E H$, McKee MD. Outcomes following plate fixation of fractures of both bones of the forearm in adults. $J$

\section{CONCLUSION}

The surgical approaches - volar approach (Henry) and dorsal approach (Thompson) were comparable in terms of functional outcome, intraoperative parameters, complications and time to union. Though cases with PIN palsy were seen in both the groups, the difference in the occurrences was not statistically significant. As both the approaches were associated with a low rate of complications, either can be used based on the preference of the operating surgeon. However, large scale multicenter studies are necessary to recommend guidelines on the choice of surgical approach for proximal radius fractures in adults.

\section{ACKNOWLEDGEMENT}

We would like to express our heartfelt gratitude to Professor Dr. Kiran Prasad Rijal for his invaluable Bone Joint Surg Am. 2007;89(12):2619-24.

8. Warwick D. Injuries of the forearm and wrist. In: Solomon L, Warwick D, Nayagam S, editors. Apley's system of orthopaedics and fractures. 9th ed: Hodder Arnold; 2010. p. 767-86.

9. Eglseder WA. Shaft fractures of the radius and ulna. In: Eglseder WA, editor. Atlas of upper extremity trauma: Springer; 2018. p. 585-642.

10. Anderson LD, Sisk D, Tooms RE, Park WI, 3rd. Compression-plate fixation in acute diaphyseal fractures of the radius and ulna. J Bone Joint Surg Am. 1975;57(3):287-97.

11. Jockel CR, Zlotolow DA, Butler RB, Becker EH. Extensile surgical exposures of the radius: a comparative anatomic study. J Hand Surg Am. 2013;38(4):745-52.

12. Henry AK. Exposures of long bones and other surgical methods: J. Wright and Sons, Limited; London, Simpkin, Marshall, Hamilton, Kent and Company, Limited; 1927.

13. The forearm. In: Hoppenfeld S, deBoer P, Buckley $\mathrm{R}$, editors. Surgical exposures in orthopaedics: the anatomic approach. 4th ed: Lippincott Williams \& Wilkins; 2009. p. 147-81.

14. Dashe J, Murray B, Tornetta P, Grott K, Mullis B, Bellevue $\mathrm{KD}$, et al. Comparison of the Henry versus Thompson approaches for fixation of proximal radial shaft fractures: A multicenter study. Paper presented at: Annual Meeting of the Orthopaedic Trauma Association (OTA); 2016 Oct 7; National Harbor, MD.

15. Thompson JE. Anatomical methods of approach in operations on the long bones of the extremities. Ann Surg. 1918;68(3):309.

16. Mekhail AO, Ebraheim NA, Jackson WT, Yeasting RA. 
Vulnerability of the posterior interosseous nerve during proximal radius exposures. Clin Orthop Relat Res. 1995(315):199-208.

17. Spinner M. Injuries to the major branches of peripheral nerves of the forearm. Plast Reconstr Surg. 1980;65(4):529.

18. Cross JD, White JA, Johnson AE, Blair JA, Hsu JR. Comparison of dorsal and volar approaches to the proximal radius. Orthopedics. 2011;34(2):93.

19. Nasab SAM, Sarrafan N, Fakoor M, Mohammadzadeh M. Comparison of volar and dorsal approaches for surgical treatment in fracture of proximal half of the radius. Pak J Med Sci. 2013;29(2):532-5.

20. Bartonicek J, Stehlik J, Jehlicka D. Plate fixation in fractures of the proximal half of the shaft of the radius. Acta Chir Orthop Traumatol Cech. 1995;62(2):86-93.

21. Kwasny O, Fuchs M, Schabus R. Results of a volar approach to plate osteosynthesis of radius shaft fractures. Theoretical basis-clinical results. Unfallchirurgie. 1992;18(1):24-30.

22. Mekhail AO, Ebraheim NA, Jackson WT, Yeasting RA. Anatomic considerations for the anterior exposure of the proximal portion of the radius. J Hand Surg Am. 1996;21(5):794-801.

23. Nasab SAM, Sarrafan N, Arti H, Aliabadi G. Outcome of forearm shaft fractures in adults treated by open reduction and internal fixation with Dynamic Compression Plate (DCP). Pak J Med Sci. 2012;28(1):45.

24. Spinner RJ, Berger RA, Carmichael SW, Dyck PJ, Nunley JA. Isolated paralysis of the extensor digitorum communis associated with the posterior (Thompson) approach to the proximal radius. J Hand Surg Am. 1998;23(1):135-41.

25. Dietz SO, Muller LP, Gercek E, Hartmann F, Rommens PM. Volar and dorsal mid-shaft forearm plating using DCP and LC-DCP: interference with the interosseous membrane and forearm-kinematics. Acta Chir Belg. 2010;110(1):60-5. 